\title{
Locally Estimated Hemodynamic Response Function and Activation Detection Sensitivity in Heroin-Cue Reactivity Study
}

\author{
Somayeh Maleki-Balajoo ${ }^{1}$, Gholam-Ali Hossein-Zadeh ${ }^{2,3}$, Hamid Soltanian-Zadeh $^{2,3,4^{*}}$, Hamed Ekhtiari \\ 1. Department of Biomedical Engineering, Faculty of Electrical Engineering, Khaje Nasir Toosi University of Technology, Tehran, Iran. \\ 2. Control and Intelligent Processing Center of Excellence, School of Electrical and Computer Engineering, University of Tehran, Tehran, Iran. \\ 3. School of Cognitive Sciences, Institute for Research in Fundamental Sciences, Tehran, Iran. \\ 4. Image Analysis Laboratory, Henry Ford Health System, Detroit, Michigan, USA. \\ 5. Research Center for Cellular and Molecular Imaging, Tehran University of Medical Sciences, Tehran, Iran. \\ 6. Iranian National Center for Addiction Studies, Tehran University of Medical Sciences, Tehran, Iran
}

Citation: Maleki-Balajoo, S., Hossein-Zadeh, G. A., Soltanian-Zadeh, H., \& Ekhtiari, H. (2016). Locally estimated hemodynamic response function and activation detection sensitivity in heroin-cue reactivity study. Basic and Clinical Neuroscience, 7(4), 299-314. http://dx.crossref. org/10.15412/J.BCN.03070403

: http://dx.crossref.org/10.15412/J.BCN.03070403

Article info:

Received: 22 April 2016

First Revision: 13 May 2016

Accepted: 25 August 2016

Key Words:

functional Magnetic

Resonance Imaging

(fMRI), Parcellation,

Hemodynamic Response

Function (HRF), Cue

reactivity, Heroin

\section{A B S T RA C T}

Introduction: A fixed hemodynamic response function (HRF) is commonly used for functional magnetic resonance imaging (fMRI) analysis. However, HRF may vary from region to region and subject to subject. We investigated the effect of locally estimated HRF (in functionally homogenous parcels) on activation detection sensitivity in a heroin cue reactivity study.

Methods: We proposed a novel exploratory method for brain parcellation based on a probabilistic model to segregate the brain into spatially connected and functionally homogeneous components. Then, we estimated HRF and detected activated regions in response to an experimental task in each parcel using a joint detection estimation (JDE) method. We compared the proposed JDE method with the general linear model (GLM) that uses a fixed HRF and is implemented in FEAT (as a part of FMRIB Software Library, version 4.1).

Results: 1) Regions detected by JDE are larger than those detected by fixed HRF, 2) In group analysis, JDE found areas of activation not detected by fixed HRF. It detected drug craving a priori "regions-of-interest" in the limbic lobe (anterior cingulate cortex [ACC], posterior cingulate cortex $[\mathrm{PCC}]$ and cingulate gyrus), basal ganglia, especially striatum (putamen and head of caudate), and cerebellum in addition to the areas detected by the fixed HRF method, 3) JDE obtained higher Z-values of local maxima compared to those obtained by fixed HRF.

Conclusion: In our study of heroin cue reactivity, our proposed method (that estimates HRF locally) outperformed the conventional GLM that uses a fixed HRF.

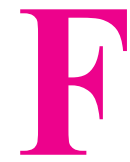

\section{Introduction}

unctional magnetic resonance imaging

(fMRI) measures small metabolic changes happening in active regions of the brain. It is a common diagnostic method for evaluating functions of a normal or diseased brain, as well as identifying neurophysiological basis of psychological and related physiological phenom-

* Corresponding Author:

Hamid Soltanian-Zadeh, PhD

Address: Control and Intelligent Processing Center of Excellence, School of Electrical and Computer Engineering, University of Tehran, Tehran, Iran Tel: +98 (21) 61114909

E-mail: hszadeh@ut.ac.ir 
ena. fMRI is based on the increase in blood flow to the local cerebral vessels that accompanies neural activity of the brain (Frackowiak et al., 2004; Jezzard, Matthews, \& Smith, 2001). The objective of fMRI data analysis is to discriminate weak blood oxygen level dependent (BOLD) signals from noisy data and determine activated regions of the brain. This process consists of two stages: preprocessing and statistical analysis.

The first stage is usually carried out in several steps such as realignment, motion correction, spatial normalization, and spatial smoothing (Frackowiak et al., 2004). Modelbased statistical analysis is mostly achieved through using general linear regression model (GLM) (Bishop, 2007; Friston et al., 1995). At the end of the learning process, the spatial map of statistical analysis coefficients resulting from the regression step is thresholded for significance (usually with a P-value over a T or F-statistics), displaying the importance of each voxel, and finally, labeling the suprathreshold regions as activated brain areas, and often overlaying them with color on an (higher resolution) anatomical MR image.

The most important issues for within-subject analyses are as follows: 1) activation detection, 2) estimation of underlying hemodynamic response, and these two issues are considered consecutively. On the other hand, localization of activated brain regions is influenced greatly by HRF model. However, HRF shape is not fixed all over the brain regions and may vary. A significant drawback of the standard GLM approach is its need to a specific assumption about the HRF. In other words, it considers a fixed HRF for the whole brain. This assumption may not be appropriate when the HRF varies from subject to subject or from region to region (Aguirre, Zarahn, \& D'Esposito, 1998; Handwerker, Ollinger, \& D'Esposito, 2004). Some methods in the literature consider a local hemodynamic response function. Spatial characteristics of fMRI are usually taken into account by estimating HRF locally. The spatial properties of hemodynamic response function are assessed by using mass-univariate methods (Friston, Jezzard, \& Turner, 1994; Friston et al., 1995; Friston \& Buchel, 2003) or multivariate pattern analysis, which assumes that the temporal behavior of HRF is the same all over the brain regions. However, based on evidence, the HRF has differences across brain regions and subjects (Aguirre et al., 1998; Handwerker et al., 2004). To address this issue, a parcellation approach can be applied as a preprocessing step to define spatially connected and functionally homogeneous components in the brain. Then, the underlying dynamics, HRF, in each homogeneous region can be estimated locally.
Parcellation concept was introduced by Flandin et al. (2002a) in order to overcome the drawback of spatial normalization. In their study, a comparison between a voxel based technique and a region based technique was done to evaluate their effects on activation detection sensitivity in fMRI data analysis. They defined parcels as spatially connected components by using anatomical data via K-means clustering method. Thus, the resulted parcellation is defined by anatomical resolution and functional images are oversampled at that resolution for further analysis. Flandin et al. (2002b) extended their previous work by defining functionally homogenous and anatomically connected parcels.

In this work, we propose a clustering approach based on Gaussian mixture models (GMMs) that group voxels from pooled multi-subject datasets according to spatial-functional features while it is blind to the subject. Also, this is the first work being proposed to overcome the anatomical and functional variability for multi-subject analysis; however, it has a few limitations. First, spatially connected feature is not achieved for all parcels and there is no guarantee that all parcels are connected spatially. Also, the resulted parcellation is not a multi-subject parcellation because all parcels are not present in each subject of the group.

It should be an important feature for any approach which is proposed to deal with anatomical and functional variability for multi-subject analysis. However, we think that the latter issue cannot be considered as a drawback because previous studies defiantly showed that HRF varies from subject to subject or even from region to region (Aguirre et al., 1998; Handwerker et al., 2004). Therefore, existence of all functionally homogenous parcels in each subject seems to be an unrealistic assumption. All mentioned limitations were addressed in Thirion et al. (2006) study in which a novel multi-subject parcellation based on spectral clustering was proposed to cope with them. Also, a hierarchical method was introduced there in order to derive group parcels that are spatially coherent and functionally homogeneous across subjects.

Another important challenge in parcellation procedure is to define the number of parcels. The precise setting of optimal number of parcels is based on using model selection approach (Thyreau, Thirion, Flandin, \& Polin, 2006). In this work, the authors claim that the optimal number of parcels was about 200 per hemisphere to have the best functional homogeneity according to the Bayesian Information Criterion (BIC). It should be mentioned that fMRI protocol used in that study was designed to activate brain areas related to several cognitive function (motor, audio, video perception, sentence analysis and computation). Thus, obtaining this number of optimal parcels, 400 , cannot be expected in fMRI data set involved in single cognitive task. 
In our study, we used the joint detection-estimation (JDE) approach proposed by Makni et al. (2008) in which the underlying dynamics, HRF, in a homogeneous region (known as a parcel throughout the paper) is estimated while activated brain regions in response to an experimental task are detected in each parcel simultaneously.

Our contribution to this paper is two-fold. First, we propose a new probabilistic based method for brain parcellation to divide brain regions into specific parcels with two characteristics. Each parcel is spatially connected and functionally homogeneous. The principle of the proposed method is very similar to Flandin, Penny, Ayache, and Poline (2003), however with some differences For one thing, parcellation is an essential preprocessing step before extracting HRF, because fMRI signals have low SNR. This estimation is difficult at the scale of one voxel so that one needs to spatially aggregate signals.

However, the extent of this spatial aggregation has to be limited because the HRF is known to fluctuate across brain regions (Handwerker et al., 2004). These considerations lead to a functional brain parcellation where a single HRF may represent each parcel. Next, estimation of HRF and detection of activations in response to an experimental task is done in each parcel according to the JDE method (Makni et al., 2008). Secondly, we investigate the effect of locally estimated hemodynamic response function on activation detection sensitivity as compared to the fixed HRF method under GLM framework which is implemented in FEAT version 5.98 (as a part of FMRIB Software Library, version 4.1, mentioned at http://www.fmrib.ox.ac.uk/fsl). To this end, we ran a study on drug-cue reactivity among heroin dependents. The real fMRI data set used in this study was provided by the Iranian National Center for Addiction Studies. These data were recorded to evaluate neural correlates of drug-cue reactivity in Heroin users. This test was designed for inducing craving using visual signs that cause craving.

This study is important as drug abuse is a significant global public health problem and is considered the main reason for continuous drug usage despite all of its adverse reactions and the main cause of relapse and treatment failure. A small yet growing number of studies have compared drug addicts and normal controls in their brain responses to drugrelated cues. Managing drug craving is central to all addiction treatment strategies. Therefore, many studies have tried to understand neural basis of craving. Previous functional neuroimaging studies have shown the involvement of prefrontal cortex (Bonson et al., 2002; Grant et al., 1996; Sell et al., 2000), limbic system (Bonson et al., 2002; Grant et al., 1996; Olbrich et al., 2006), basal ganglia (nucleus accumbens, striatum [putamen and caudate nucleus], globus pallidus, and the subthalamic nucleus) (Breiter et al., 1997; Brody et al., 2007; Kilts et al., 2001; Myrick, Anton, \& Li, 2004; Olbrich et al., 2006), and other subcortical structures (Bonson et al., 2002; Grant et al., 1996; Olbrich et al., 2006) during presentation of drug-related cues to drug-dependent subjects. Multiple corresponding regions of interests (ROIs) exist in drug-cue reactivity, but inconsistent results in different studies with the conventional analysis method, encouraged us to select "drug-cue reactivity" as our target field. In this paper, we proposed local estimation of HRF and evaluated its benefit in identifying brain regions involved in heroin cue reactivity compared to the use of a fixed, global HRF.

The rest of the paper is organized as follows. In section 2, we explain details of the proposed procedure. Section 3 describes the fMRI data acquisition and reports the performance of our approach using synthetic and real fMRI datasets. Finally, section 4 presents discussion and conclusions of the work.

\section{Methods}

We proposed a method in probabilistic framework for brain parcellation. In this method, brain is divided into spatially connected and functionally homogeneous parcels by using both anatomical and functional features of the fMRI data. This new representation of the fMRI data is useful in region-based analysis, especially in local estimation of HRF. In this regard, in each parcel, the joint estimationdetection approach developed by Makni et al. (2008) was applied to estimate local HRFs and identify activated areas. To this end, active regions were identified by modeling active and nonactive voxels as a mixture model. By modeling voxel activities through the mixture model in a Bayesian framework, the posterior distributions of all model parameters were calculated. Then, these parameters were estimated by Markov Chain Monte Carlo method from their posterior distributions. Via calculation of model parameters for each parcel, an estimate of the hemodynamic response function and activated brain regions were obtained.

\subsection{Parcellation}

\subsubsection{Extracting anatomic and functional features of the brain}

As we indicated in previous section, each parcel has two specific characteristics; being spatially connected component and functionally homogeneous. Thus, the voxels of a parcel are interconnected and have similar time courses or homogeneous activities (which could be summarized by some model parameters). We proposed a parcellation ap- 
proach that considers both aforementioned characteristics. This algorithm relied on the optimization of a compound criterion reflecting both the spatial and functional structures of the brain. Three spatial coordinates of each voxel were used in the model as the anatomical information. Also, functional properties of each voxel was brought into the model by the estimated $\beta$-parameters resulted from applying GLM analysis on time series data via:

$$
y=X \beta+e
$$

where $y$ is the observed time-series, $\mathrm{X}$ is the designed matrix composed of a set of basic functions, $\beta$ is the vector of parameters (regression weights of the basis function in the designed matrix), and e is the residual error.

The $\beta$ parameters model the behavior of each voxel in response to the stimulus pattern and generate a d-dimensional feature vector for each voxel as $\bar{\beta}_{v}=\left(\beta_{1}, \ldots, \beta_{d}\right)$. The final feature vectors are calculated as $\left(\bar{f}_{v}\right)_{v=1: V}=\left[\bar{\tau}_{v}, \bar{\beta}_{v}\right]$ , where represents a voxel and the number of feature vectors/voxels. All these feature vectors come from one of the $\mathrm{K}$ classes, here modeling features from different parcels. Feature vectors drawn from the class $\mathrm{k}$ follow the probability density function (pdf), $f_{k}\left(v \mid \theta_{k}\right) K=1 \ldots . k$ where $f_{k}$ is a parametric form of a probability density function with the vector of unknown parameters.

A Gaussian mixture model (GMM) was fitted to this data. Thus, the parametric form of the $\operatorname{pdf} f_{k}$ is known but the value of the parameter vector $\theta_{k}$ is unknown. Each class has a prior $\mathrm{w}_{\mathrm{k}} \varepsilon[0,1]$ probability, expressing the fraction of the feature vectors following the density $f_{k}$. These mixing parameters, $w_{k}$, satisfy the following condition:

$$
\sum_{k=1}^{K} w_{k}=1
$$

The values of $w_{k}$ are initially unknown. As Figure 1 demonstrates, the data (the set of feature vectors) may be described by a finite mixture model (FMM) through the following equation:

$$
\mathrm{P}(\mathrm{F}(\mathrm{v})=\mathrm{F} \mid \Theta, \mathrm{K})=\sum_{\mathrm{K}-1}^{\mathrm{K}} \mathrm{w}_{\mathrm{k}} \mathrm{N}\left(\mu_{\mathrm{k}}, \sum_{\mathrm{k}}\right)
$$

, where $\mathrm{F}$ is the set of feature vectors, while is the set of unknown parameters, including the means and covariance of the classes and the weights:

$$
\Theta=\left\{w_{1} \ldots . w_{k-1}, \mu_{1}, \ldots, \mu_{k}, \sum_{1, \ldots . .,} \sum_{k}\right\}
$$

Given the feature vectors as $f_{v}: v=1, \ldots . v$, we use the maximum likelihood (ML) method to estimate. To this end, the following optimization problem must be solved.

$$
\begin{aligned}
& \Theta=\operatorname{Arg} \max _{\Theta} L(\Theta) \\
& \Theta=\operatorname{Arg} \max _{\Theta} \sum_{v=1}^{v} \log f\left(f_{v} \mid \Theta\right)
\end{aligned}
$$

After estimating the parameters and fitting the GMM model, the data were clustered by the Bayes classifier according to the following relation (Tohka et al., 2007) where the class $L_{v}$ of the voxel $v$ is:

$$
L_{v}=\operatorname{argmax}_{\left.K \varepsilon_{1}^{\prime}, \ldots, K\right\}} w_{k} N\left(f_{v} ; \mu_{k}, \sum_{k}\right), v=1, \ldots \ldots, V
$$

If the labels of the classes, $L_{v}: v=1, \ldots ., V$ were independent, the Bayes classifier (along with the assumption of correct component densities) would lead to the lowest classification error.

Expectation-maximization (EM) algorithm is a method for finding maximum likelihood estimates of the parameters. The EM algorithm can be a best solution for GMM if the number of components is known ahead, and the initialization is close to the true parameter values. To address the above requirements, we used FMM and Akaike information criterion (AIC). Therefore, we proposed the following method that assumes an FMM for distribution of the feature vectors in which the statistical distributions of voxels are assumed to be Gaussian in each class.

\subsubsection{Expectation maximization algorithm with self- annealing behavior}

Here, we briefly explain the EM algorithm proposed by Figueiredo with self-annealing behavior and high entropy initialization property (Figueiredo \& Jain, 2000). Feature vectors, which include both anatomical and functional properties of voxels extracted from fMRI data are fed into EM algorithm. Distribution of these vectors is assumed to be a FMM, and the EM algorithm fits this model on the data. Initialization strategy, which can be interpreted as a self-annealing behavior, makes this approach superior to the GMM (with standard EM algorithm).

The EM algorithm and its application to Gaussian FMMs are well known, and hence this subsection is intentionally concise. The EM algorithm produces a sequence of parameter estimates, $\{\Theta(t) \mid t=0,1, \ldots .$.$\} , by alternately applies the$ following two steps until convergence.

E-Step: The conditional expectation of the log likelihood $1(\Theta)$ given the data and the current parameter estimate $\Theta(t)$ 


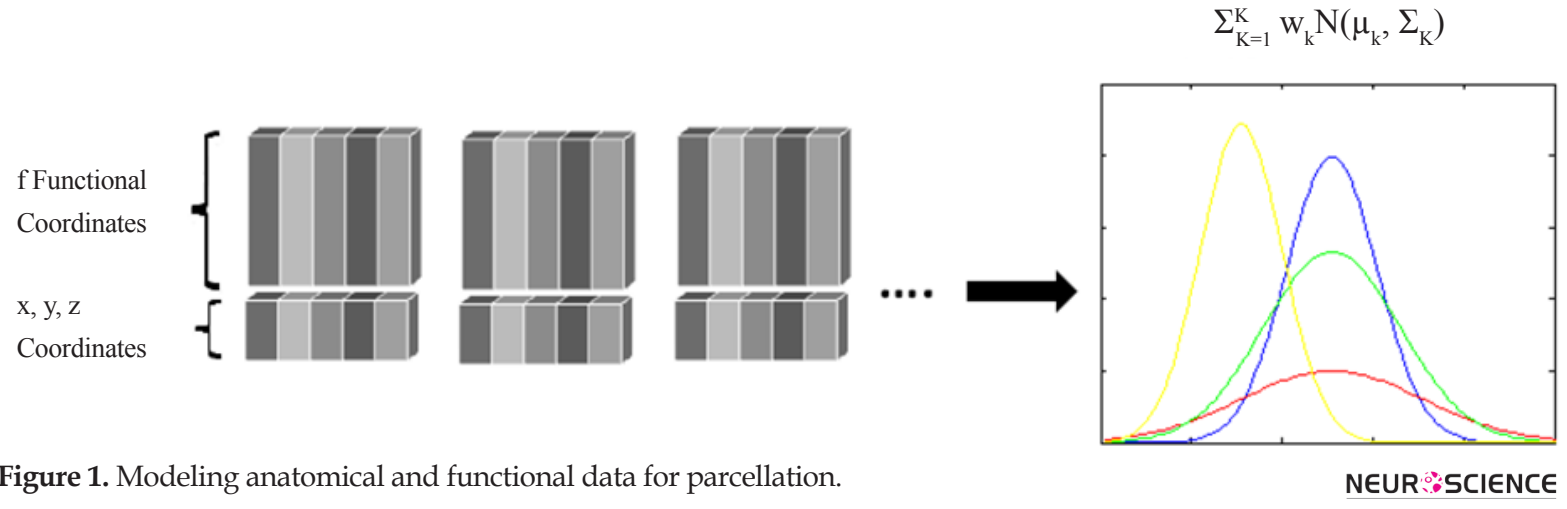

are computed. In the case of FMMs, it is reduced to compute the posterior probabilities:

$$
J_{v k}(t)=p\left(k \mid \Theta(t), f_{v}\right)
$$

$J_{v k}(t)$ is calculated for each data point, $f_{v}$ and class $k$.

M-step: Updates of the parameter vector are computed by maximizing (9):

$$
Q(\Theta \mid \Theta(t))=\sum_{v=1}^{v} \sum_{k=1}^{K} J_{v k}(t)\left[\log w_{k}+\log f_{k}\left(f_{v} \mid \Theta\right)\right](9)
$$

We apply a "high entropy initialization" which leads to self-annealing behavior as explained by Tohka et al. (2007). The EM algorithm is initialized by setting, where $e_{v k}$ is a random perturbation parameter:

$$
\begin{aligned}
& J_{v k}(0)=1 / K+e_{v k} \\
& v=1, \ldots ., v \\
& K=1, \ldots ., K
\end{aligned}
$$

The initial posteriors are used to compute the initial values of the parameter vectors. In other words, both the posterior probabilities and initial values are resulted in the E-step. Actually, the initial value of the parameter vector obtained in the M-step is used to compute in the E-step. Also, the initial value of mean and covariance will be close to the sample mean and covariance of the data (fv).

Thus, the entropy of the posterior probabilities of all voxels are maximized by setting them nearly equal to all classes. This initialization procedure is called high entropy initialization (Ueda \& Nakano, 1998). As this value is a stationary point of the EM algorithm, it cannot be selected equally.

The high entropy initialization causes EM algorithm to behave as the deterministic annealing in which the thermo- dynamical free energy cost function is minimized. In this optimization problem, simulated annealing avoids getting caught in suboptimal maxima because of its stochastic counterpart (Tohka et al., 2007).

\subsubsection{Determining the number of parcels}

The number of parcels, $\mathrm{K}$, is an important parameter in the algorithm. The precise setting of optimal number of parcels is based on using model selection approach (Thyreau et al., 2006). To compare mixtures with different numbers of components, various criteria have been developed. These include $\mathrm{AIC}$, the Bayesian information criterion, and the minimum message length criterion. In most applications, the EM algorithm is randomly initialized for $K=1, \ldots ., K_{\max }$, where $K_{\max }$ is some prespecified maximum number of components. The algorithm is run for different values of $\mathrm{K}$ and the criterion is evaluated. Then, the model that achieves the minimum value of the criterion is selected. A main problem with this technique is the selection of the maximum number of components. If it is too small, the model may be too coarse for the data; if it is too large, the computational time may take very long time.

In this work, we employ AIC to fit the model to the data. The algorithm is run for each $\mathrm{K}$ and the criterion is evaluated. Then, the $\mathrm{K}$ value that achieves the minimum amount is selected as the number of parcels. The larger the number of parcels, the higher the degree of within-parcel homogeneity. However, there exists a trade-off between the withinparcel homogeneity and the signal-to-noise ratio (SNR).

\subsubsection{AIC model selection}

The objective of AIC model selection is to estimate the information loss when the probability distribution "f" associated with the true (generating) model is approximated by probability distribution " $\mathrm{g}$ " associated with the model that is to be evaluated (Wagenmakers \& Simon, 2004). The Kullback-Leibler information quantity is used as a measure 
for difference between the true model and its approximation. Akaike has shown that a model Mi that has the lowest AIC value is asymptotically equivalent to choosing a model that minimizes the expected Kullback-Leibler discrepancy. The AIC is defined as:

$$
A I C_{i}=-2 \log L_{i}+2 V_{i}
$$

This formula computes the maximum likelihood for the entrant model "i." This parameter is specified by setting the free parameter. These parameters were determined in a way that to maximize the probability of the entrant model which generated the observed data.

In this equation, descriptive accuracy is rewarded by the maximum likelihood and lack of parsimony is penalized according to the number of free parameters. Note that Equation 11 is valid only for sufficiently large data sets. The finite sample correction is generally recommended when $\mathrm{n} / \mathrm{V}>40$ (Tohka et al., 2007).

$$
\mathrm{ALC}_{\mathrm{c}}=-2 \log L+2 V+{ }^{2 V(V+1)} /_{(n-v-1)}
$$

The above criterion can also be described as an entropy concept. This criterion is presented based on the relative measure of loss when the desired model is applied on the data. We use this concept of AIC criterion in our work. Appropriate model selection process based on this criterion regards that AIC criterion is calculated for each model according to the following equations:

$$
\begin{aligned}
& \mathrm{RSS}=\sum_{\mathrm{i}=1}^{\mathrm{n}} \dot{\varepsilon}_{\mathrm{i}}^{2} \\
& \mathrm{AlC}=2 \mathrm{k}+\mathrm{n}\left[1 \mathrm{n}^{(2 \pi R S S)} / \mathrm{n}+1\right]
\end{aligned}
$$

In the above equations, $\mathrm{V}$ is the number of parameters in the statistical model, $\mathrm{n}$ is the total number of observations, and RSS is the residual sum of squares. The model that allocates the lowest AIC is chosen as the optimal model. In the case of determining the number of parcels, the algorithm runs for different values of $\mathrm{K}$ and in each case, the value of $\mathrm{AIC}$ is calculated. Ultimately, the optimal $\mathrm{K}$ is the one that minimizes AIC.

\subsection{Parcel-based detection of activation regions} and estimation of hemodynamic response function

After segregating the brain into spatially connected and functionally homogeneous parcels, active regions were identified in each parcel by modeling active and non-active voxels as a mixture. The time invariant model of the BOLD signal introduced by Makni et al. (2008) was used to account for voxel-dependent and task-related BOLD response of each voxel. In this model, each parcel is characterized by a single HRF shape and an activation magnitude for each voxel and stimulus type. Therefore, the HRF shape was assumed constant within a parcel, while the magnitude of the activation could vary in space and across experimental conditions.

$$
y_{j}=\sum_{m=1}^{M} a_{j}^{m} X^{m} h+P l_{j}+b_{j}
$$

In Equation 15, refers to the BOLD fMRI time course measured in each voxel, is a binary matrix corresponding to the arrival times for the $\mathrm{m}^{\text {th }}$ stimulus, $\mathrm{h}$ denotes the unknown HRF shape in each parcel, is the activation magnitude in each voxel, $\mathrm{P}$ is the low frequency orthogonal matrix, denotes the set of low frequency drifts involved in each parcel, and is the noise vector in each voxel.

By modeling voxel activities through the mixture model in the Bayesian framework, the posterior distributions of all model parameters were calculated. Then, these parameters are estimated by the Markov chain Monte Carlo (MCMC) method from their posterior distributions. By calculating the model parameters in each parcel, estimates of the hemodynamic response function and activated brain areas in that region are obtained. A comprehensive description of the joint activation estimation method can be found in Makni et al. (2008) study.

\section{Results}

\subsection{Synthetic fMRI dataset/parcellation}

We evaluated the accuracy of our proposed parcellation method using synthetic data. The fMRI data were generated by adding activations to a resting state fMRI dataset that included noise, signal drift, and other realistic parameters. The achieved results of the proposed algorithm were compared with previous developed methods such as K-means and Gaussian mixture model (GMM). Thus, we assessed the confusion matrix and the accuracy (AC) parameter based on the results of the synthetic functional data.

In designing simulated dataset, we considered some totally distinct regions and some regions with overlaps. Therefore, 4 different regions (Two of them were completely distinct but the other regions had overlaps) were specified in the brain (Figure 2). Then, specific time-series were created by convolving 2 hypothetical stimulus patterns with different hypothetical hemodynamic responses for each region. These time-series (activations) were added to the time-series of voxels in 4 regions. 


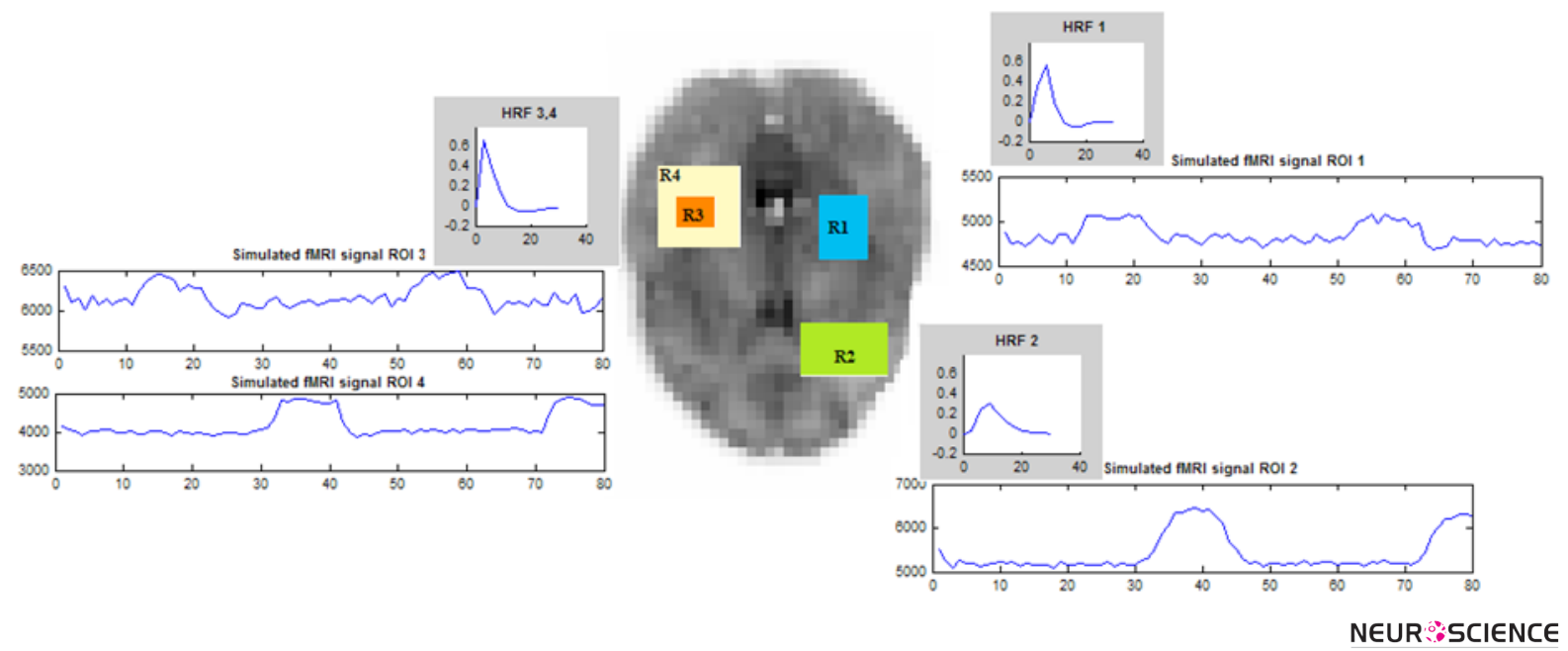

Figure 2. Synthetic fMRI dataset for parcellation: 4 different regions (R1, R2, R3, and R4) are specified in the brain. R1 and R2 are completely distinct but R3 and R4 are overlapped.

The AC parameter is defined as the proportion of the correct detections (sum of true positives and true negatives) to the total number of voxels. This parameter shows the efficiency of the classification. Comparison of the results shows that our proposed method outperforms the other ones and has the highest AC (Table 1).

\subsection{Experimental fMRI dataset}

Besides proposing a new parcellation method used as a prestep of the JDE approach, we developed an fMRI study to explore specific patterns of brain activities in cue-induced craving in heroin abusers in order to examine the effect of variable HRF, estimated locally in each parcel, versus the fixed HRF on activation detection sensitivity.

\subsubsection{Participants}

In this section, the data under analysis are briefly described. Thirty male active injection heroin users, fulfilling the DSM-IV diagnostic criteria for opioid dependence (Diagnostic and Statistical Manual of Mental Disorders, Fourth Edition, American Psychiatric Association, 1994), aged 2842, and native Persian speakers with normal vision were enrolled. All subjects were strongly right-handed as indicated by a handedness inventory. The subjects were selected among treatment seeker patients from the waiting list of methadone maintenance clinics in Iranian National Center for Addiction Studies (INCAS) after receiving their signed informed consent forms. Their participation in this study was prior to receiving any treatment. Their main opiate consumption pattern was intravenous injection for at least the last 6 months. The Ethics Committee of the University of Tehran Medical Sciences approved the study protocol.

\subsubsection{Pictorial heroin-cue induced craving assessment task}

Heroin-related stimuli were selected from a previously evaluated photograph series prepared by Ekhtiari, Behzadi, Oghabian, Edalati, and Mokri (2006). These pictures consisted of the most craving-induced images such as hands holding syringe, powder of heroin, injecting syringe, and scenes of drug preparation for injection. Sixteen heroin-related and 16 neutral images were presented to each subject in a block design with 4 blocks in total ( 8 pictures in each block) as the fMRI task. After each activation block, a white fixation cross on a black background was presented for 24 seconds (Figure 3 ).

The neutral stimuli were derived from the neutral schema pictures with the same geometry, color, and background of the stimuli pictures. Examples of the stimuli and neutral pictures presented during the fMRI scan are shown in Figure 3. After the imaging session, all participants were tested for the level of craving with visual analog scale (VAS). To this end, craving inducing pictures of the fMRI task were used in printed for-

Table 1. Comparison of the algorithms performance using the accuracy (AC) parameter.

\begin{tabular}{|c|c|c|c|}
\hline Method & FMM-EM algorithm self-annealing & K-means & $\begin{array}{c}\text { Gaussian Mixture Model } \\
\text { (GMM) }\end{array}$ \\
\hline Accuracy (AC) & 0.98 & 0.84 & 0.63 \\
\hline
\end{tabular}



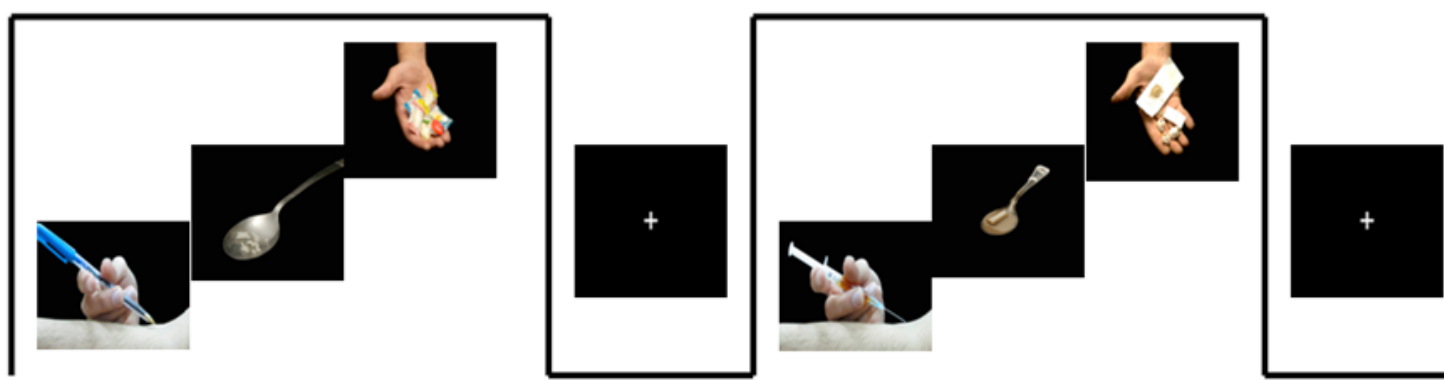

Figure 3. Functional imaging study of pictorial heroin-cue-induced craving assessment task.

NEUR:SIENCE

mat to develop a task consisting of 16 craving inducing cues and 2 neutral pictures. Each picture was shown to the subjects by interviewer for 10 seconds in a fixed order (same order as the fMRI task but each 8 craving pictures followed by only 1 neutral picture). The subjects were asked to quantify their inner craving feeling after watching each picture with a VAS ruler $(20 \mathrm{~cm}$ line from "not at all" to "at most" with 1 to 100 grades on its back). The mean scores of VASs for $16 \mathrm{crav}$ ing pictures were assigned as subjective cue-induced
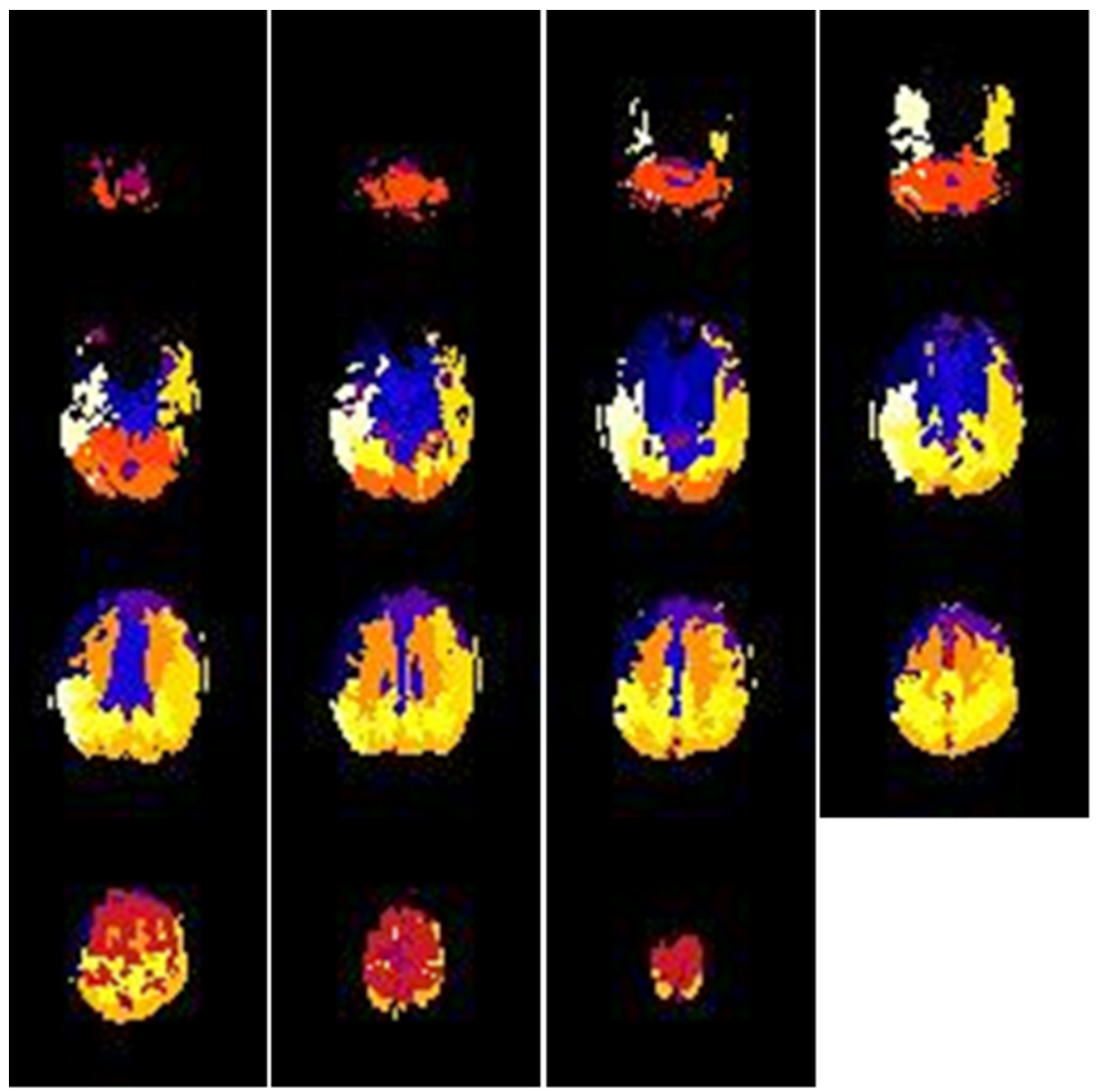

NEUR:SCIENCE

Figure 4. Proposed parcellation approach results for one subject, divided to 13 functionally homogeneous areas based on AIC criterion. craving (CIC) score. The recorded scores of VAS from 0 to 100 were categorized in 3 subdivisions: non-responder (VAS:0-30), semi-responder (VAS:30-70), and responder (VAS:70-100) (Ekhtiari et al., 2007). Finally, 12 out of 30 subjects turned out to be responders whose data were used in this study.

\subsubsection{Data acquisition}

We used a 1.5 Tesla GE Signa scanner and the fMRI data were obtained using an Echo Planar Imaging (EPI) proto- 
col $(\mathrm{TE}=60 \mathrm{~ms}, \mathrm{TR}=3000 \mathrm{~ms}$, slice thickness $=7 \mathrm{~mm}$, band width $=62.5 \mathrm{KHz}$, and flip angle $=90^{\circ}$ ). Fifteen contiguous axial slices, parallel to the "anterior commissure-posterior commissure" line were acquired according to the Talairach atlas. 3D T1-weighted images were also obtained for registration of fMRI data to the structural map of the brain.

\subsubsection{Data analysis}

FEAT package (fMRI Expert Analysis Tool) version 5.42 (obtained from http://www.fmrib.ox.ac.uk/fsl) was used for data preprocessing. At the first step of preprocessing, motion correction using FMRIB's linear registration tool (MCFLIRT) (Jenkinson \& Smith, 2001) was applied. Then, non-brain areas were excluded by using FMRIB's brain extraction tool (BET) (Smith, 2002). Afterwards, spatial smoothing with a Gaussian kernel of $5 \mathrm{~mm}$ FWHM, intensity normalization, and high-pass temporal filtering were used in preprocessing pipeline. However, the filtering could not reduce signal fluctuations synchronous with the breathing cycle (because breathing cycles occur at about 0.3 $\mathrm{Hz}$, and not to be sampled with a TR of $3 \mathrm{~s}$ ). However, it would reduce signal variations related to breath-to-breath changes in respiration depth and rate (Gaussian weighted least squares straight line fit with sigma equal to $25 \mathrm{~s}$ ). This filter is a sharp roll off FIR filter that did not introduce autocorrelations to the data. Time-series statistical analysis was carried out using FMRIB's improved linear model (FILM) with local autocorrelation correction (Woolrich, Ripley, \& Brady, \& Smith, 2001).

Heroin cues versus neutral pictures modeled as explanatory variables in both methods, because our objective in this fMRI study was identifying brain regions specially involved in cue-reactivity procedure. These variables were used for statistical analysis within the context of the general linear mode. Z-values (Gaussianized T/F) of statistical images were thresholded using voxels determined by $\mathrm{Z}>2.3$ and a (corrected) voxel significance threshold of P-value equal to 0.05 . To analyze these datasets, 4 desired contrasts were defined: 1) neutral, 2) craving, 3) neutral $>$ craving, and 4) neutral $<$ craving.
After data preprocessing, the functional features of the voxels were achieved by the estimated $\beta$-parameters for the 4 desired contrasts. After combining these features with the corresponding anatomical features that include the coordinates of each voxel, the desired data were ready for parcellation.

\subsubsection{Obtained results}

As stated previously, the purpose of analyzing the above data with the JDE method is to probe the effect of locally estimated HRF on the results of activation detection versus considering a fixed HRF for the whole brain. In Figure 4, the result of the proposed parcellation approach on a subject is shown which is divided to 13 functionally homogeneous areas based on AIC. In order to determine the number of parcels, AIC values for $\mathrm{K}=1: 30$ was calculated and the optimal model (corresponding to minimum AIC) was found to be $\mathrm{K}=13$. In previous work, the optimal number of parcels was about 200 per hemisphere in order to have the best functional homogeneity according to Bayesian information criterion (Thyreau et al., 2006).

It should be mentioned that fMRI protocol used in that study was designed to activate brain areas related to several cognitive functions (motor, audio, video perception, sentence analysis, and computation). Thus, it cannot be expected to obtain this number of optimal parcels, 400, in fMRI data set involved in single cognitive task like the task we used in this experiment. Moreover, our dataset was acquired with block-design protocol and variety of hemodynamic response function was less reflected in this kind of dataset compared to the event-related designed dataset.

To better represent the results of parcellation, masks of a parcel out of 13 parcels are shown in Figure 5. Integration and coherence of voxels within each parcel are shown in Figure 5. After defining functionally homogenous parcels, the JDE method was applied on the data to simultaneously estimate HRF and activated voxels in each parcel. To evaluate the effect of locally estimated HRF on the activation detection sensitivity, the results of this method were compared to the results of standard GLM method using a fixed HRF.
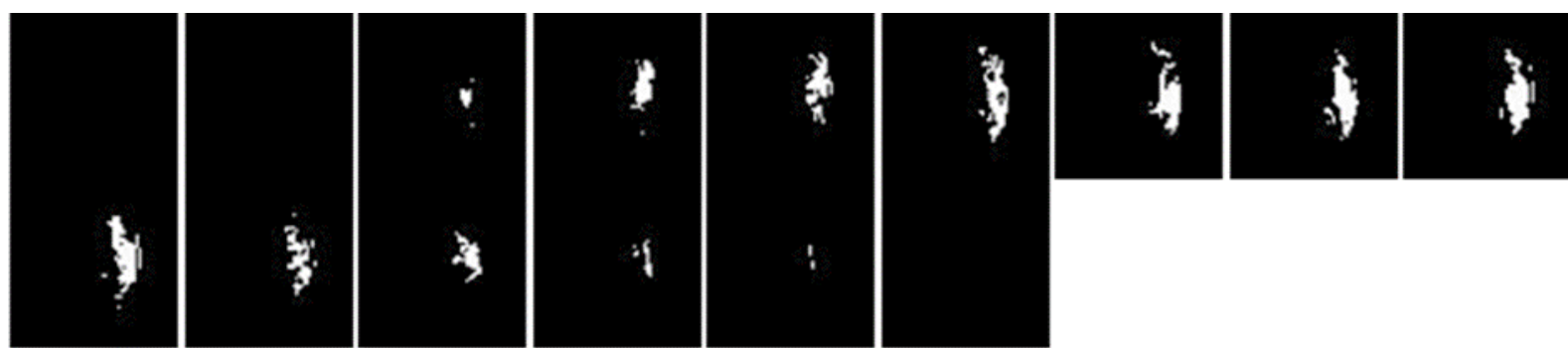

Figure 5. One parcel out of the 13 parcels, shown in Figure 4

NEUR 
Thresholded activation images $\quad 2.3-11.0$

Group Analysis on optimal contrast (Craving $>$ Neutral) _._._. standard GLM method with fix HRF

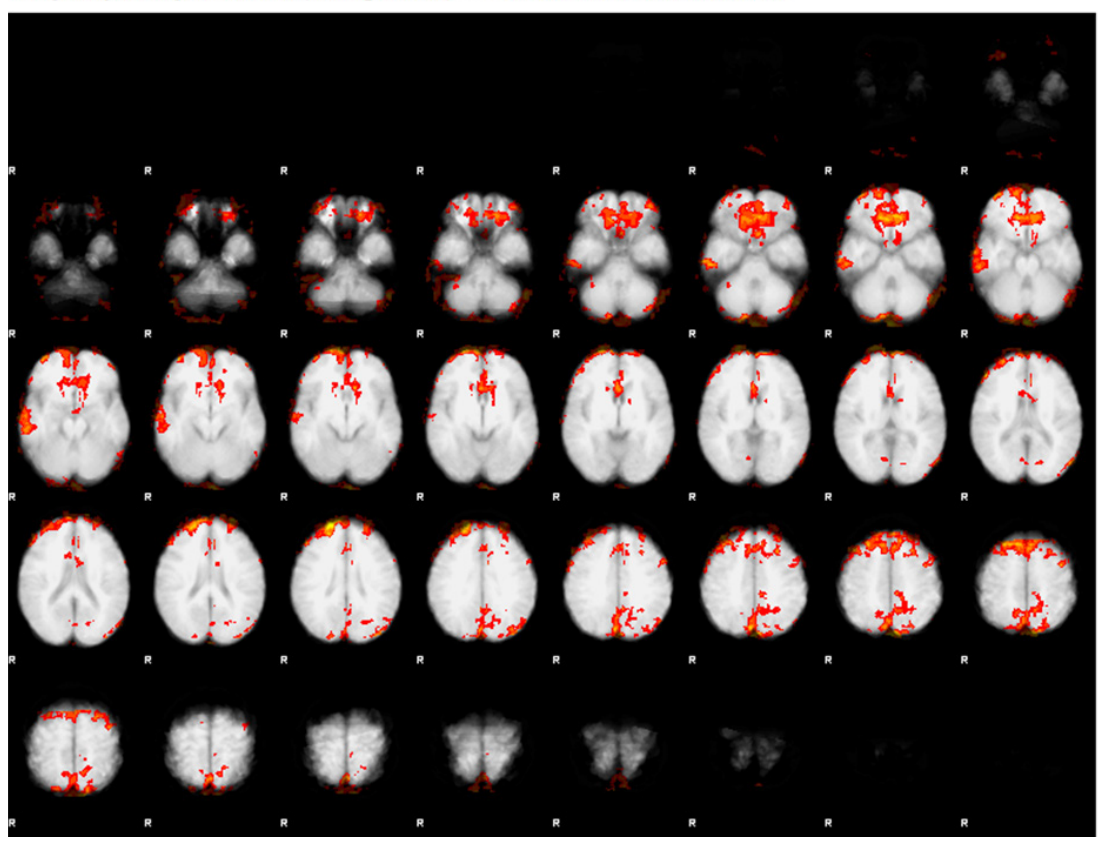

NEUR:SCIENCE

Figure 6. The average of group analysis on 12 patients by the FSL software for optimal contrast (craving $>$ neutral).

Likewise, to evaluate the effect of using variable HRF versus fixed $\mathrm{HRF}$ and reliability of activated areas in the heroin-cue reactivity study, group analysis was con- ducted based on the Bayesian mixed effects analysis method for both approaches. The optimal contrast, craving $>$ neutral, was selected among the 4 contrasts, since

Thresholded activation images $\quad 2.3=11.4$

Group Analysis on optimal contrast (Craving $>$ Neutral) ___ JDE method with locally variable HRF

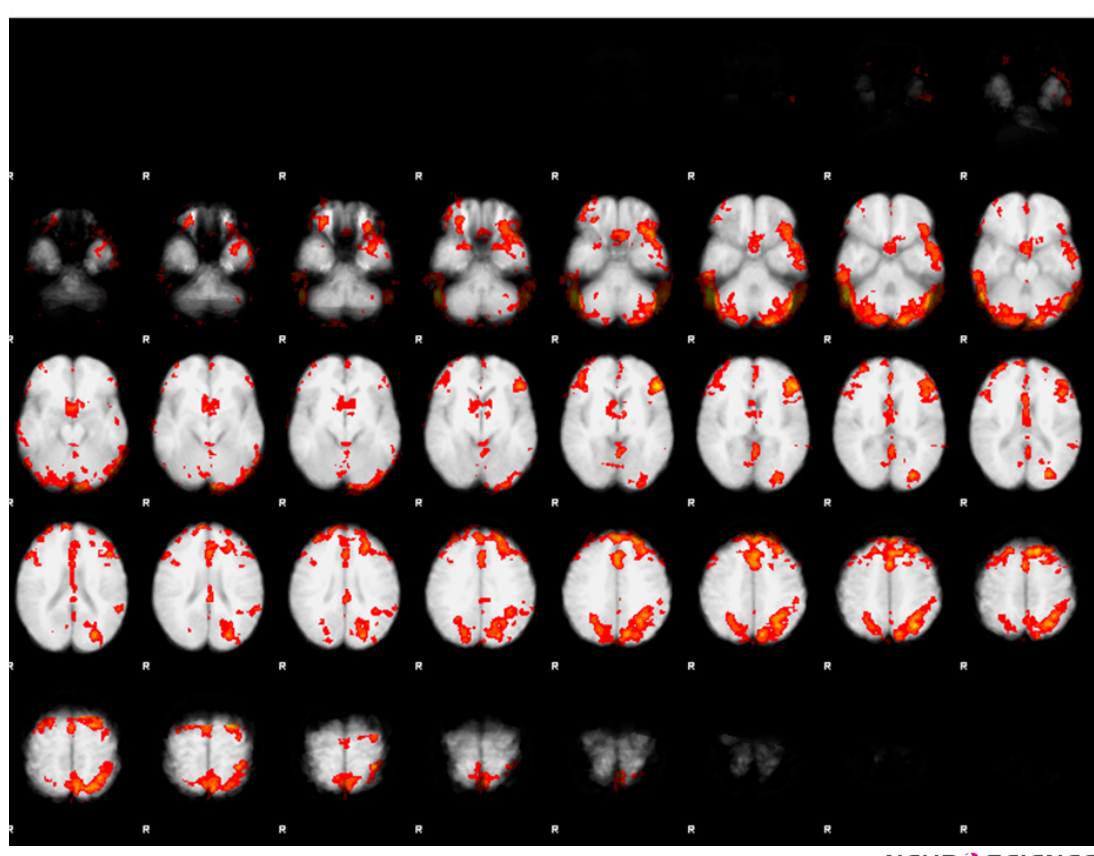

NEUR:SCIENCE

Figure 7. The average of group on 12 patients by joint detection estimation method based on functionally homogenous regions for optimal contrast (craving>neutral). 
this contrast provides useful information regarding brain regions involved in heroin-cue reactivity study. The group analysis results are illustrated in Figures 6 and 7, respectively, for the standard GLM and JDE methods.
Finally, to evaluate the achievements of JDE method based on locally HRF estimation in functionally homogenous regions, the activated areas were reported based on specific region of interest (ROI). To this end, functional brain maps

Table 2. Comparison and analysis of the obtained results of both methods by calculating the percentage of activated voxels and $\mathrm{Z}_{\text {max }} / \mathrm{Z}_{\text {mean }}$ for each regain in left and right hemisphere.

\begin{tabular}{|c|c|c|c|c|c|c|c|c|c|c|}
\hline & \multirow[b]{3}{*}{$\begin{array}{c}\text { Sub- } \\
\text { corti- } \\
\text { cal } \\
\text { area }\end{array}$} & \multirow[b]{3}{*}{ Talarich ROI } & \multicolumn{4}{|c|}{ JDE method, HRF is estimated locally } & \multicolumn{4}{|c|}{ Standard GLM, a fixed HRF } \\
\hline & & & \multicolumn{2}{|c|}{ Left hemisphere } & \multicolumn{2}{|c|}{ Right hemisphere } & \multicolumn{2}{|c|}{ Left hemisphere } & \multicolumn{2}{|c|}{ Right hemisphere } \\
\hline & & & $\begin{array}{l}\text { Percent- } \\
\text { age of } \\
\text { activated } \\
\text { voxels } \\
\text { (\%) }\end{array}$ & $\begin{array}{l}Z_{\text {max }} / \\
z_{\text {mean }}\end{array}$ & $\begin{array}{l}\text { Percent- } \\
\text { age of } \\
\text { activated } \\
\text { voxels } \\
\text { (\%) }\end{array}$ & $Z_{\text {max }} / Z_{\text {mean }}$ & $\begin{array}{l}\text { Percent- } \\
\text { age of } \\
\text { activated } \\
\text { voxels } \\
\text { (\%) }\end{array}$ & $\begin{array}{l}z_{\text {max }} / \\
z_{\text {mean }}\end{array}$ & $\begin{array}{l}\text { Percent- } \\
\text { age of } \\
\text { activated } \\
\text { voxels } \\
\text { (\%) }\end{array}$ & $\mathrm{Z}_{\text {max }} / \mathrm{Z}_{\text {mean }}$ \\
\hline 1 & \multirow{6}{*}{$\begin{array}{l}\text { Cere- } \\
\text { bellum }\end{array}$} & $\begin{array}{l}\text { Posterior lobe. } \\
\text { tuber }\end{array}$ & 47.96 & $8.61 / 4.35$ & 40.68 & $11.44 / 5.30$ & 37.68 & $8.46 / 4.47$ & 24.52 & $6.53 / 3.72$ \\
\hline 2 & & $\begin{array}{l}\text { Anterior lobe. } \\
\text { culmen }\end{array}$ & 5.31 & $3.82 / 2.74$ & 2.95 & $5.26 / 2.95$ & & & 10.13 & $7.64 / 3.96$ \\
\hline 3 & & $\begin{array}{l}\text { Posterior lobe. } \\
\text { declive }\end{array}$ & 50.09 & $9.08 / 4.19$ & & & 10.46 & $8.53 / 4.70$ & & \\
\hline 4 & & $\begin{array}{c}\text { Posterior lobe. } \\
\text { uvula }\end{array}$ & 22.55 & $6.00 / 3.09$ & 10.54 & $4.40 / 3.09$ & & & & \\
\hline 5 & & $\begin{array}{l}\text { Posterior lobe. } \\
\text { pyramis }\end{array}$ & & & & & 13.18 & $7.36 / 4.16$ & 15.88 & $8.23 / 4.23$ \\
\hline 6 & & $\begin{array}{l}\text { Inferior semi- } \\
\text { lunar lobule }\end{array}$ & & & & & 8.91 & $5.56 / 3.30$ & & \\
\hline 7 & \multirow{2}{*}{$\begin{array}{l}\text { Basal } \\
\text { ganglia }\end{array}$} & $\begin{array}{c}\text { Sublobar. caudate } \\
\text { head }\end{array}$ & 60.34 & $6.77 / 4.20$ & 53.81 & $4.89 / 3.33$ & 32.32 & $5.67 / 3.29$ & & \\
\hline 8 & & $\begin{array}{l}\text { Sublobar. puta- } \\
\text { men }\end{array}$ & 16.33 & $7.00 / 4.93$ & 30.76 & $4.144 / 3.10$ & 15.59 & $5.94 / 2.99$ & & \\
\hline 9 & \multirow{8}{*}{$\begin{array}{l}\text { Limbic } \\
\text { lobe }\end{array}$} & $\begin{array}{l}\text { Limbic lobe. } \\
\text { cingulate gyrus }\end{array}$ & 27.74 & $6.93 / 3.93$ & 18.72 & $7.29 / 4.00$ & & & & \\
\hline 10 & & $\begin{array}{c}\text { Limbic lobe. } \\
\text { cingulate gyrus. } \\
\text { BA32 }\end{array}$ & 27.67 & $8.80 / 4.23$ & 36.98 & $7.24 / 4.21$ & 31.5 & $8.77 / 4.49$ & & \\
\hline 11 & & $\begin{array}{l}\text { Limbic lobe. ante- } \\
\text { rior cingulate }\end{array}$ & 18.36 & $5.49 / 3.50$ & 19.93 & $5.83 / 3.664$ & 41.15 & $7.11 / 3.69$ & 37.9 & $7.77 / 3.85$ \\
\hline 12 & & $\begin{array}{l}\text { Limbic lobe. } \\
\text { anterior cingulate. } \\
\text { BA24 }\end{array}$ & & & & & 34.22 & $5.05 / 3.35$ & 45.57 & $6.25 / 3.53$ \\
\hline 13 & & $\begin{array}{l}\text { Limbic lobe. } \\
\text { anterior cingulate. } \\
\text { BA3 }\end{array}$ & & & & & & & 16.52 & $8.61 / 5.21$ \\
\hline 14 & & $\begin{array}{l}\text { Limbic lobe. pos- } \\
\text { terior cingulate. } \\
\text { BA23 }\end{array}$ & 69.41 & $5.28 / 3.55$ & & & & & & \\
\hline 15 & & $\begin{array}{l}\text { Limbic lobe. pos- } \\
\text { terior cingulate }\end{array}$ & 59.11 & $5.72 / 3.86$ & & & & & & \\
\hline 16 & & $\begin{array}{c}\text { Limbic lobe. } \\
\text { cingulate gyrus. } \\
\text { BA23 }\end{array}$ & 68.96 & $5.04 / 3.68$ & & & & & & \\
\hline
\end{tabular}




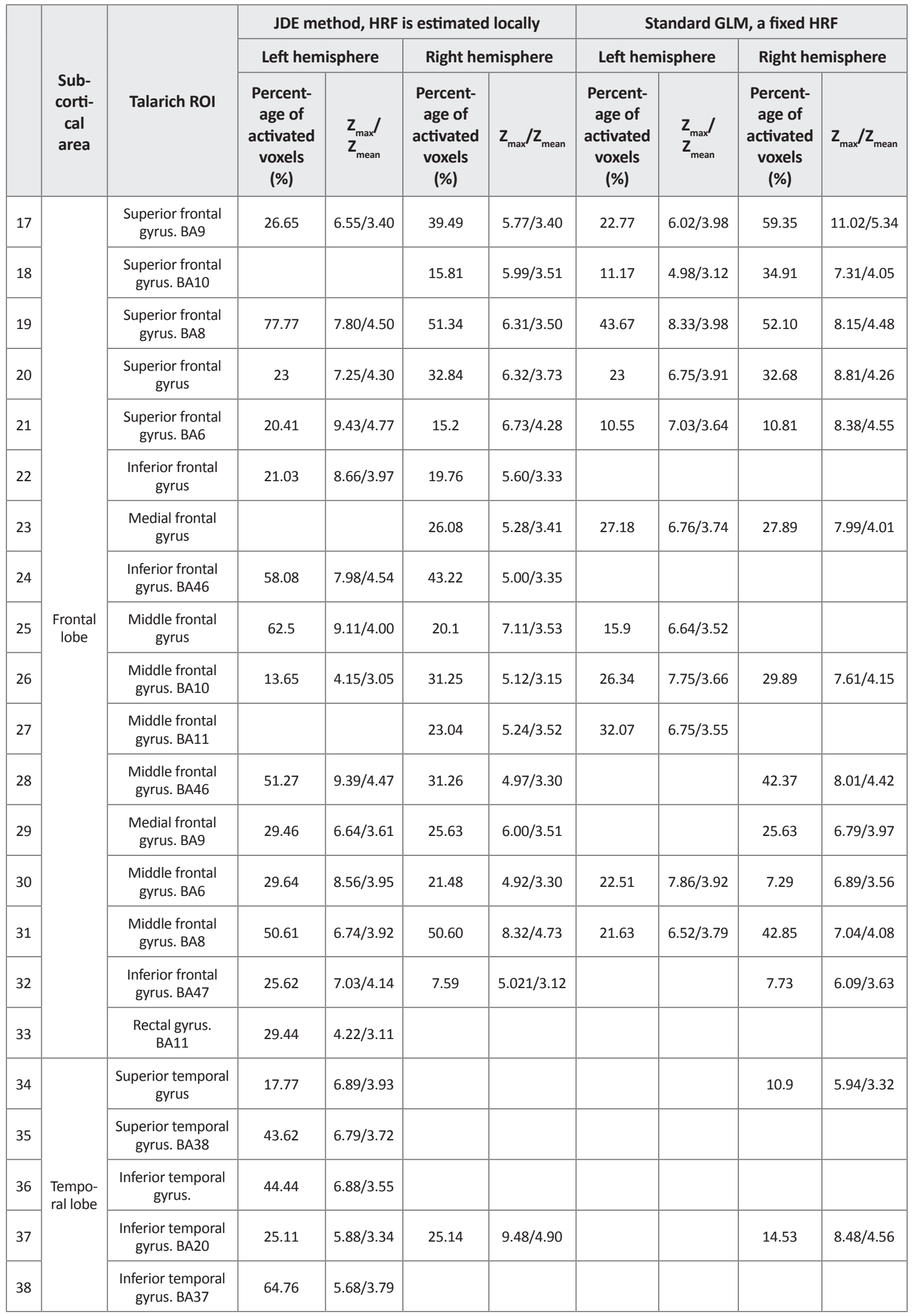




\begin{tabular}{|c|c|c|c|c|c|c|c|c|c|c|}
\hline & \multirow[b]{3}{*}{$\begin{array}{c}\text { Sub- } \\
\text { corti- } \\
\text { cal } \\
\text { area }\end{array}$} & \multirow[b]{3}{*}{ Talarich ROI } & \multicolumn{4}{|c|}{ JDE method, HRF is estimated locally } & \multicolumn{4}{|c|}{ Standard GLM, a fixed HRF } \\
\hline & & & \multicolumn{2}{|c|}{ Left hemisphere } & \multicolumn{2}{|c|}{ Right hemisphere } & \multicolumn{2}{|c|}{ Left hemisphere } & \multicolumn{2}{|c|}{ Right hemisphere } \\
\hline & & & $\begin{array}{l}\text { Percent- } \\
\text { age of } \\
\text { activated } \\
\text { voxels } \\
\text { (\%) }\end{array}$ & $\begin{array}{l}\mathrm{Z}_{\text {max }} / \\
\mathrm{Z}_{\text {mean }}\end{array}$ & $\begin{array}{l}\text { Percent- } \\
\text { age of } \\
\text { activated } \\
\text { voxels } \\
\text { (\%) }\end{array}$ & $Z_{\text {max }} / Z_{\text {mean }}$ & $\begin{array}{l}\text { Percent- } \\
\text { age of } \\
\text { activated } \\
\text { voxels } \\
\text { (\%) }\end{array}$ & $\begin{array}{l}\mathrm{Z}_{\text {max }} / \\
\mathrm{Z}_{\text {mean }}\end{array}$ & $\begin{array}{l}\text { Percent- } \\
\text { age of } \\
\text { activated } \\
\text { voxels } \\
\text { (\%) }\end{array}$ & $\mathrm{Z}_{\text {max }} / \mathrm{Z}_{\text {mean }}$ \\
\hline 39 & \multirow{4}{*}{$\begin{array}{l}\text { Tempo- } \\
\text { ral lobe }\end{array}$} & $\begin{array}{l}\text { Middle temporal } \\
\text { gyrus }\end{array}$ & 16.28 & $6.48 / 4.19$ & & & 19.08 & $6.19 / 3.54$ & 18.67 & $6.59 / 4.22$ \\
\hline 40 & & $\begin{array}{c}\text { Middle temporal } \\
\text { gyrus. BA } 21\end{array}$ & 27.6 & $6.40 / 3.35$ & 11.13 & $6.86 / 4.51$ & & & 28.95 & $7.49 / 4.30$ \\
\hline 41 & & Fusiform gyrus & & & 38.34 & $9.69 / 5.04$ & & & & \\
\hline 42 & & $\begin{array}{c}\text { Fusiform gyrus. } \\
\text { BA } 37\end{array}$ & 36.90 & $7.55 / 4.75$ & 44.44 & $11.42 / 5.74$ & & & & \\
\hline
\end{tabular}

(suprathreshold regions as activated brain areas were overlaid on higher resolution anatomical MRI) resulting from group analysis in the two methods were registered to a Talarich atlas template. Then, the Talarich atlas index template was used to automate labeling of the activated areas, for the ultimate goal of finding the number of activated voxels in each Talarich ROI. Moreover, the maximum of the Z-values and their mean value in an ROI was calculated to examine the ability of the 2 methods in generating strong Z-values.

Table 2 presents a comparison and analysis of the obtained results of 2 methods. The thresholds in both methods were set to $\mathrm{Z}>2.3$ and the corrected voxel significance threshold of $\mathrm{P}$ value was 0.05 for having the same false alarm rate. The results illustrated the main achievements as follows: 1) The volume of the detected regions by JDE method was larger than the other method, 2) In the group analysis, JDE method found those activations that were missed by the fixed HRF; for example, it detected a priori "regions-of-interest" in the limbic lobe (anterior cingulate cortex [ACC], posterior cingulate cortex [PCC] and cingulate gyrus), basal ganglia, especially striatum (putamen and head of caudate) and cerebellum in addition to the areas detected by the standard GLM with fixed HRF, and 3) JDE method obtained higher Z-values of local maxima compared to the standard GLM that uses a fixed HRF. These activated brain regions are the main areas involved in cue reactivity or response to drug related cues during "craving induction by cues" paradigms. They were also reported in previous studies as the areas involved in craving (Bonson et al., 2002; Grant et al., 1996; Olbrich et al., 2006; Breiter et al., 1997; Brody et al., 2007; Kilts et al., 2001; Myrick et al., 2004).

\section{Discussion}

We presented a probabilistic method for defining the functional regions of the brain based on finite mixture model and examining the effect of locally estimated HRF on activation detection sensitivity. Also, we used JDE method to estimate hemodynamic response function locally and detected activated areas simultaneously in each functional region. Simulation studies showed that the parcellation results of our proposed method were more accurate than previous methods like K-means and GMM.

Experimental results showed that local estimation of HRF by JDE method has great impact on the reliability of the activation detection, confirming Badillo, Vincent, and Ciuciu (2011) study. In addition, they showed that the use of local HRF increased the volume of the detected areas. Also, some drug craving a priori ROI were detected in the limbic lobe (ACC, PCC, and cingulate gyrus), basal ganglia, especially striatum (putamen and head of caudate) and cerebellum. These brain regions are activated as the main areas involved in cue reactivity or response to drug-related cues during "craving induction by cues" paradigms. These areas were also reported in previous studies (Bonson et al., 2002; Grant et al., 1996; Olbrich et al., 2006; Breiter et al., 1997; Brody et al., 2007; Kilts et al., 2001; Myrick et al., 2004). Limbic system includes brain areas which provide the anatomical substrate for motivated behavior anatomically and functionally. In the study of Rodriguez de Fonseca and Navarro (1998), the functional role of main limbic system elements, especially the extended amygdala and its connections to drug dependence was reviewed. Moreover, great activation was detected in the cerebellum. In contrast to the traditional belief that cerebellum is purely a motor control device, 
apparently it also contributes to cognitive processing and emotional control (Franklin et al., 2007).

Activated areas in both methods (cited in Table 2) were largely left lateralized, which is similar to the findings among other drug users for instance, cocaine users (Garavan et al., 2000). The main component of basal ganglia is striatum (caudate nucleus and putamen). A part of striatum is connected to limbic structures such as amygdala, hippocampus, midline thalamus, and certain regions of the prefrontal cortex. The functional role of striatum is to control emotional and motivational aspects of behavior. Different forms of psychopathology such as addictive behavior cause dysfunction of striatum structurally and functionally (Everitt et al., 1999; Robbins \& Everitt, 2002).

Animal and human imaging studies have revealed the key role of striatum as a focal point for the binge/intoxication stage in the addiction cycle (Koob \& Volkow, 2010). The main function of the caudate and putamen in drug addiction is related to their participation in habit-based learning that may drive compulsive drug seeking behavior (Frederico et al., 2012). Third, the Z-values originating from the locally HRF consideration are larger than the Z-values resulted from fixed HRF consideration. The larger Z-value indicates that the activation level of that region is high due to the stimulus pattern and shows the significance of the area in task-related involvements of ROIs (details are provided in Table 2). Importance of drug craving experience in the continuation of addiction has been repeatedly confirmed. Better understanding of neural correlates of heroin craving is important both theoretically and clinically. These may suggest methods given HRF locally as a potential alternative analysis pipeline for the future drug craving fMRI studies.

\section{Acknowledgements}

The aurthors would like to thank Arian Behzadi for his significant role in clinical and psychological assesment of the subjects as well as Mohammad Ali Oghabian and Habib Ganjgahi from Research Center for Molecular and Cellular Imaging, Tehran University of Medical Sciences for their valuable assistance in image acquisitions. The data acquistion phase was funded by a grant from the Tehran University of Medical Sciences.

\section{Conflict of Interest}

All authors declared no conflict of interest.

\section{References}

Aguirre, G. K., Zarahn, E., \& D'Esposito, M. (1998). The variability of human bold hemodynamic responses. Neuroimage, $8(4)$, 360-69.

Badillo, S., Vincent, T., \& Ciuciu, P. (2011, March). Impact of the joint detection-estimation approach on random effects group studies in fMRI. Proceedings of the 8th IEEE International Symposium on Biomedical Imaging: From Nano to Macro (pp. 376-80). USA: Chicago, Illinois.

Bechara, A., Damasio, H., \& Damasio, A. (2000). Emotion, decision making and the orbitofrontal cortex. Cerebral Cortex, 10(3), 295-307.

Bishop, C. M. (2007). Pattern recognition and machine learning. New York: Springer-Verlag Press.

Bonson, K. R., Grant, S. J., Contoreggi, C. S., Links, J. M., Metcalfe, J., Weyl, H. L., et al. (2002). Neural systems and cue induced cocaine craving. Neuropsychopharmacology, 26(3), 37686

Breiter, H. C., Gollub, R. L., Weisskoff, R. M., Kennedy, D. N., Makris, N., Berke, J. D., et al. (1997). Acute effects of cocaine on human brain activity and emotion. Neuron, 19(3), 591-611.

Brody, A. L., Mandelkern, M. A., Olmstead, R. E., Jou, J., Tiongson, E., Allen, V., et al. (2007). Neural substrates of resisting craving during cigarette cue exposure. Biological Psychiatry, 62(6), 642-51

Ekhtiari, H., Behzadi, A., Oghabian, M. A., Edalati, H., \& Mokri, A. (2006). Evaluation of visual cues inducing drug craving in intravenous heroin users. Advances in Cognitive Science, 8(31), 43-51.

Ekhtiari, H., Mokri, A., Edalati, H., Safaei, H., Jannati, A., \& Razzaghi, M. E. (2007). Designing and evaluation of reliability and validity of a visual cue induced craving assessment task for intravenous heroin users. European Psychiatry, 22, S186S187.

Everitt, B. J., Parkinson, J. A., \& Olmstead, M. C., Arroyo, M., Robledo, P., \& Robbins, T. W. (1999). Associative processes in addiction and reward: the role of amygdala-ventral striatal subsystems. Annals of the New York Academy of Sciences, 877(1), 412-38.

Figueiredo, M., \& Jain, A. (2000). Unsupervised selection and estimation of finite mixture models. Paper presented at the 15th International Conference on Pattern Recognition, Barcelona, Spain, 3-7 September 2000.

Flandin, G., Kherif, F., Pennec, X., Malandain, G., Ayache, N., \& Poline, J. B. (2002a). Improved detection sensitivity of functional MRI data using a brain parcelling technique. Proceedings of the 5th International Conference on Medical Image Computing and Computer Assisted Intervention (pp. 467-74). Berlin: Springer.

Flandin, G., Kherif, F., Pennec, X., Rivie`re, D., Ayache, N., \& Poline, J. B. (2002b). Parcellation of brain images with anatomical and functional constraints for fMRI data analysis. Proceedings of the 1st International Symposium on Biomedical Imaging (pp. 907-10). New Jersey: Institute of Electrical and Electronics Engineers. 
Flandin, G., Penny, W., Pennec, X., Ayache, N., \& Poline, J. B. (2003). A multisubject anatomo-functional parcellation of the brain. Neuroimage, 19, 1600-605.

Frackowiak, R., Ashburner, J., Penny, W., Zeki, S., Friston, K., \& Frith, C. (2004). Human brain function (2nd ed.). San Diego, C.A.: Academic Press.

Frederico, C., Pereira, F. C., Viana, S., Mendes, P., Ali, S. F., \& Ribeiro, C. F. (2012). Caudate-Putamen: Dopamine homeostasis and Astrocyte activation in methamphetamine abuse. In A. Costa \& E. Villalba (Eds.), Horizons in neuroscience research (pp. 1-28). Huntington, N.Y.: Nova Silences Publication.

Franklin, T. R., Wang, Z., Wang, J., Sciortino, N., Harper, D., Li, Y., et al. (2007). Limbic activation to cigarette smoking cues independent of nicotine withdrawal: a perfusion fMRI study. Neuropsychopharmacology, 32(11), 2301-309.

Friston, K. J., \& Buchel, C. (2003). Statistical Parametric Mapping: The Analysis of Functional Brain Images. San Diego, C.A.: Academic Press.

Friston, K. J., Holmes, A. P., Poline, J. B., Frith, C. D., \& Frackowiak, R. S. J. (1995). Statistical parametric maps in functional imaging: A general linear approach. Human Brain Mapping, 2, 189-210.

Friston, K. J., Holmes, A. P., Poline, J. B., Grasby, P. J., Williams, S. C., Frackowiak, R. S., et al. (1995). Analysis of fMRI timeseries revisited. Neuroimage, 2(1), 45-53.

Friston, K. J., Jezzard, P., \& Turner, R. (1994). Analysis of functional MRI time-series. Human Brain Mapping, 1(2), 153-71.

Garavan, H., Pankiewicz, J., Bloom, A., Cho, J. K., Sperry, L., Ross, T. J., et al. (2000). Cue induced cocaine craving: Neuroanatomical specificity for drug users and drug stimuli. American Journal of Psychiatry, 157(11), 1789-798.

Grant, S., London, E. D., Newlin, D. B., Villemagne, V. L., Liu, X., Contoreggi, C., et al. (1996). Activation of memory circuits during cue elicited cocaine craving. Proceedings of the National Academy of Sciences of the United States of America, 93(21), 12040-2045.

Handwerker, D. A., Ollinger, J. M., \& D’Esposito, M. (2004) Variation of BOLD hemodynamic responses across subjects and brain regions and their effects on statistical analyses. Neuroimage, 21(4), 1639-651

Haynes, J. D., \& Rees, G. (2005). Predicting the stream of consciousness from activity in human visual cortex. Current Biology, 15(14), 1301-307.

Jenkinson, M., \& Smith, S. (2001). A global optimisation method for robust affine registration of brain images. Medical Image Analysis, 5(2), 143-46.

Jezzard, P., Matthews, P. M., \& Smith, S. M. (2001). Functional MRI: An introduction to methods. New York: Oxford University Press.

Kilts, C. D., Schweitzer, J. B., Quinn, C. K., Gross, R. E., Faber, T. L., Muhammad, F., et al. (2001). Neural activity related to drug craving in cocaine addiction. Archives of General Psychiatry, 58(4), 334-41.

Koob, G. F., \& Volkow, N. D. (2010). Neurocircuitry of addiction. Neuropsychopharmacology, 35(1), 217-38.
Makni, S., Idier, J., Vincent, T., Thirion, B., Dehaene-Lambertz, Gh., \& Ciuciu, P. (2008). A fully Bayesian approach to the parcel-based detection-estimation of brain activity in FMRI Neuroimage, 41(3), 941-69.

Myrick, H., Anton, R. F., Li, X. X., Henderson, S., \& George, M. (2004). Differential brain activity in alcoholics and social drinkers to alcohol cues: relationship to craving. Neuropsychopharmacology, 29(2), 393-402.

Olbrich, H. M., Valerius, G., Paris, C., Hagenbuch, F., Ebert, D., \& Juengling, F. D. (2006). Brain activation during craving for alcohol measured by positron emission tomography. Austral ian and New Zealand Journal of Psychiatry, 40(2), 171-78.

Robbins, T. W., \& Everitt, B. J. (2002). Limbic-striatal memory systems and drug addiction. Neurobiology of Learning and Memory, 78(3), 625-36.

Rodríguez de Fonseca, F., \& Navarro, M. (1998). Role of the limbic system in dependence on drugs. Annals of Medicine, 30(4), 397-405.

Sell, L. A., Morris, J. S., Bearn, J., Frackowiak, R. S., Friston, K J., \& Dolan, R. J. (2000). Neural responses associated with cue evoked emotional states and heroin in opiate addicts. Drug and Alcohol Dependence, 60(2), 207-16.

Smith, S. M. (2002). Fast robust automated brain extraction. $\mathrm{Hu}-$ man Brain Mapping, 17(3), 143-55.

Thirion, B., Flandin, G., Pinel, P., Roche, A., Ciuciu, P., \& Poline, J. B. (2006). Dealing with the shortcomings of spatial normalization: Multi-subject parcellation of FMRI datasets. Human Brain Mapping, 27(8), 678-93.

Thyreau, B., Thirion, B., Flandin, G., \& Polin, J. B. (2006, ). Anatomo-functional Description of the brain: A Probabilistic approach Paper presented at the 31th IEEE International Conference on Acoustics, Speech, and Signal Processing, Toulouse, France, 14-19 May 2006.

Tohka, J., Krestyannikov, E., Dinov, I. D., Mackenzie Graham A., Shattuck, W., Ruotsalainen, U., et al. (2007). Genetic algorithms for Finite Mixture Model based voxel classification in neuroimaging. IEEE Transactions on Medical Imaging, 26(5), 696-711.

Ueda, N., \& Nakano, R. (1998). Deterministic annealing EM algorithm. Neural Networks, 11(2), 271-82.

Volkow, N. D., Wang, G. J., Fowler, J. S., Hitzemann, R., Angrist, B., Gatley, S. J., et al. (1999). Association of methylphenidateinduced craving with changes in right striato-orbitofrontal metabolism in cocaine abusers: implications in addiction American Journal of Psychiatry, 156(1), 19-26.

Volkow, N. D., Wang, G. J., Ma, Y., Fowler, J. S., Wong, C., Ding, Y. S., et al. (2005). Activation of orbital and medial prefrontal cortex by methylphenidate in cocaine-addicted subjects but not in controls: relevance to addiction. Journal of Neurosciences, 25(15), 3932-939.

Wagenmakers, E. J., \& Simon, F. (2004). Notes and CommentAIC model selection using Akaike weights. Psychonomic Bulletin \& Review, 11(1), 192-96.

Wang, Z., Faith, M., Kerrin, K., Tang, K., Wileyto, E. P., Patterson, F., et al. (2007). Neural substrates of abstinence induced cigarette cravings in chronic smokers. Journal of Neurosciences, 27(51), 14035-4040 
Woolrich, M. W., Ripley, B. D., Brady, M., \& Smith, S. M. (2001). Temporal autocorrelation in univariate linear modeling of FMRI data. Neuroimage, 14(6), 1370-386.

Worsley K. J., \& Friston, K. J. (1995). Analysis of FMRI time series revisited again. Neuroimage, 2(3), 173-81. 